
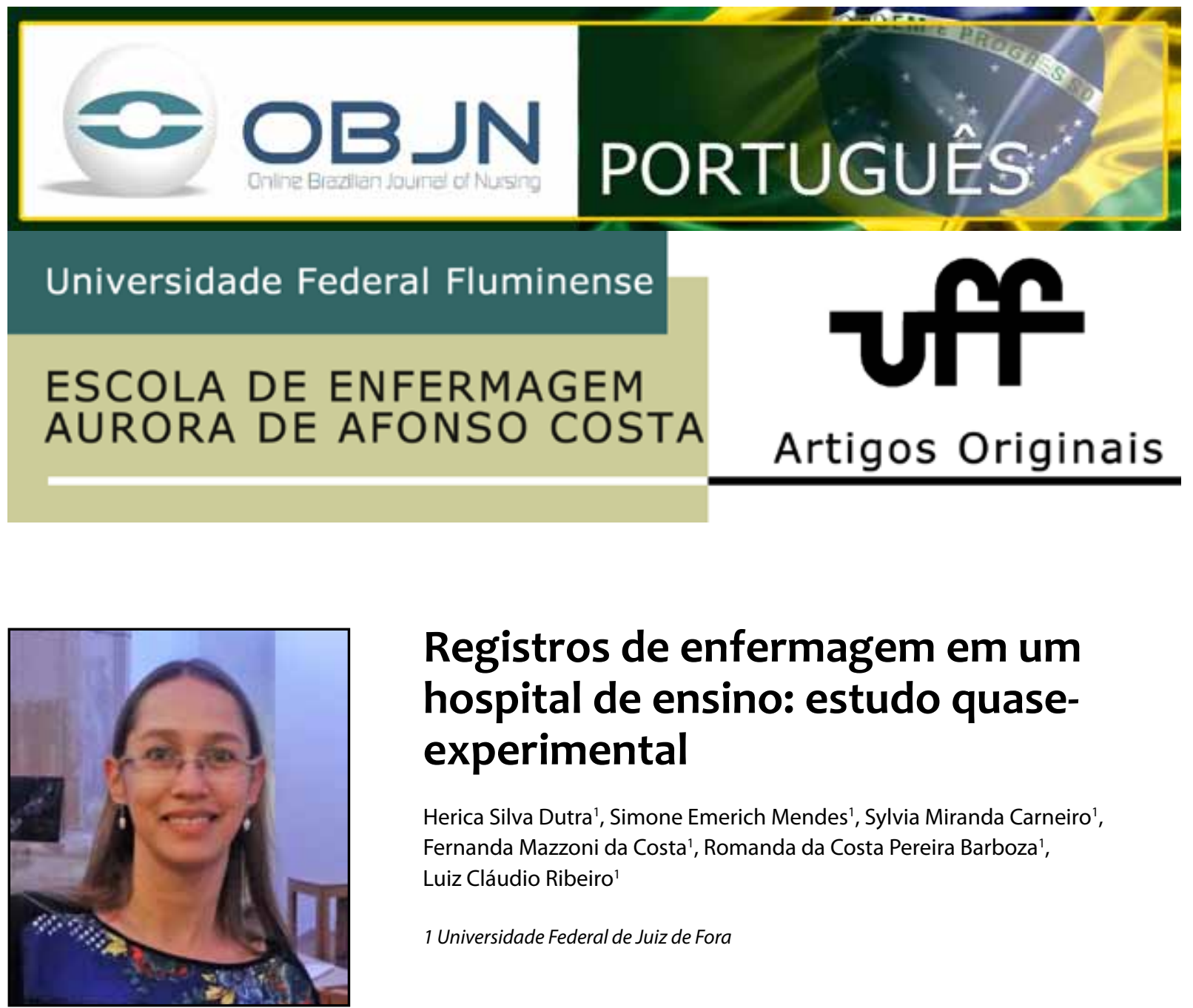

\title{
Registros de enfermagem em um hospital de ensino: estudo quase- experimental
}

\author{
Herica Silva Dutra', Simone Emerich Mendes', Sylvia Miranda Carneiro', \\ Fernanda Mazzoni da Costa', Romanda da Costa Pereira Barboza', \\ Luiz Cláudio Ribeiro \\ 1 Universidade Federal de Juiz de Fora
}

\section{RESUMO}

Objetivo: avaliar os registros da equipe de enfermagem quanto ao cumprimento da legislação específica em um hospital de ensino considerando-se o setor da instituição, o turno de trabalho, a categoria profissional e o número de infrações por registro antes e após intervenção educativa. Método: estudo quase-experimental do tipo pré-teste e pós-teste com um único grupo. Resultado: foram avaliados 826 registros de enfermagem. O maior número de registros foi feito na Unidade de Terapia Intensiva, pelos técnicos de enfermagem e no turno noturno. Houve um aumento na quantidade de registros feitos pelos auxiliares de enfermagem nos setores clínica masculina e cirurgia feminina. Houve redução quanto à ocorrência de três infrações ou mais. A intervenção foi eficaz para os itens presença de data, hora, assinatura e carimbo. Discussão: anotações incompletas podem dificultar a assistência individualizada, prejudicar a continuidade da assistência e implicar riscos para a segurança do paciente. Conclusão: intervenções educativas podem contribuir para melhorias nos registros de enfermagem.

Descritores: Registros de Enfermagem; Enfermagem; Estudos de Intervenção; Hospitais de Ensino; Equipe de Enfermagem. 


\section{INTRODUÇÃO}

Os registros de enfermagem são parte integrante do sistema de informação nas instituições de saúde e são considerados um importante instrumento de comunicação(1). São realizados pela equipe de enfermagem com a finalidade de fornecer informações sobre o estado geral do paciente, possibilitando a comunicação entre a equipe de enfermagem e, também, a equipe multiprofissional. Os dados descritos registram a continuidade da assistência, respaldando legalmente o trabalho do profissional, além de servir de instrumento para auditorias, validam a prática profissional e são utilizados como fonte de investigação científica, auxiliando nas pesquisas e no ensino(2) ${ }^{(2)}$ Além disso, as informações registradas pelos profissionais de saúde são fonte de dados para a tomada de decisões gerenciais, devendo tais dados serem completos e fidedignos ${ }^{(3)}$.

O prontuário é o acervo de todos os documentos relacionados ao cuidado prestado ao paciente. Nele, estão contidas informações fornecidas pela equipe multiprofissional responsável pelos cuidados a esse paciente, sendo um instrumento de comunicação entre todos os membros da equipe de saúde ${ }^{(4)}$. Os registros de enfermagem fazem parte do prontuário do paciente e servem de suporte documental das atividades desenvolvidas pelos profissionais de enfermagem. Seu principal objetivo é descrever a assistência de enfermagem prestada ao paciente/família, incluindo a descrição da situação de saúde/doença, o planejamento da assistência, sua implementação, as reações do paciente/família às intervenções realizadas e a avaliação dos resultados obtidos.

A equipe de enfermagem deve prever o tempo para documentar as atividades realizadas na assistência aos indivíduos/famílias no prontuário, pois fazem parte das responsabilidades legais desses profissionais que permanecem 24 horas junto ao paciente, podendo contribuir com maior quantidade de informações a respeito da assistência prestada ${ }^{(5)}$.

Cabe comentar que os registros de enfermagem são utilizados para outros fins, como: pesquisa; ensino; gestão de recursos humanos, físicos e materiais; auditoria de custos e da assistência; e reembolsos de seguradoras de saúde ${ }^{(2)}$. Ressalta-se o valor jurídico-legal do prontuário, já que os registros são provas documentais de atos profissionais ${ }^{(2,6)}$. Quando bem redigidos e detalhados, os registros de enfermagem podem afastar alegações de imperícia, imprudência ou negligência na prestação de serviços ${ }^{(5)}$.

Para que os registros de enfermagem sejam considerados documentos válidos, eles devem seguir critérios determinados pelos órgãos regulamentadores do exercício profissional de enfermagem. As informações relativas ao processo de cuidar devem ser redigidas de forma clara, correta, objetiva e organizada; devem ser livres de rasuras; constar a identificação completa do paciente e do profissional responsável por sua realização; além de identificação de data e hora referentes à informação registrada ${ }^{(7)}$. Os registros de enfermagem também são considerados como indicadores de qualidade. Por meio deles, são fornecidos dadose fatos que embasam o planejamento da assistência de enfermagem ${ }^{(1,8)}$. Com o propósito de planejar, executar e avaliar continuamente a atuação da sua equipe e do cuidado por ela prestado, o enfermeiro deve assumir seu papel de liderança a fim de promover mudanças no cenário da prática assistencial e garantir subsídios legais para a prática diária(9).

Considerando-se o que foi anteriormente exposto, adotou-se como objeto desta investigação os registros de enfermagem de uma instituição hospitalar de ensino.

A realização da presente investigação se justifica pelos seguintes argumentos quanto aos registros de enfermagem: 1) fornecem as informações necessárias para o cuidado e o acompanhamento do paciente; 2) possibilitam a comunicação 
entre os membros da equipe de enfermagem e multidisciplinar, assegurando a continuidade e a qualidade da assistência prestada; 3) são considerados evidência legal dos cuidados prestados; 4) são fonte para ensino, pesquisas e auditorias; 5) quando corretamente registrados, permitem reembolsos e justificam os custos das atividades realizadas ${ }^{(8)}$; 6) a existência e a disponibilidade de informações completas, corretas, organizadas e seguras podem reduzir ruídos na comunicação, falhas no processo de trabalho e riscos à segurança dos pacientes ${ }^{(10)}$;7) alegações de imperícia, imprudência ou negligência na assistência podem ser afastadas quando os registros de enfermagem são contínuos e atualizados ${ }^{(5)}$; 8) qualificam o cuidado e promovem o empoderamento dos profissionais de enfermagem ${ }^{(2)}$. Além disso, ressalta-se que a comunicação segura é uma das metas para a segurança do paciente nos serviços de saúde ${ }^{(11)}$.

Assim, o estudo teve como objetivo avaliar os registros da equipe de enfermagem, quanto ao cumprimento da legislação específica, em um hospital de ensino considerando-se o setor da instituição, o turno de trabalho, a categoria profissional e o número de infrações por registro antes e após intervenção educativa.

\section{MÉTODO}

Trata-se de estudo quase-experimental do tipo pré-teste e pós-teste com um único grupo acerca dos registros de enfermagem nos prontuários de pacientes de um hospital de ensino da Zona da Mata Mineira. Estudos do tipo quase-experimento são aqueles em que não é possível seguir rigorosamente os critérios de um experimento verdadeiro, como a distribuição aleatória dos sujeitos em grupo experimental e grupo controle. Porém, a introdução de manipulação ou de experimento pelo pesquisador possibilita a observação de seu efeito na variável de interesse. No caso desta investigação, o grupo controle e o grupo experimental foram formados pelos mesmos sujeitos (profissionais de enfermagem da instituição alvo). Realizou-se uma avaliação prévia e outra posterior à realização do experimento (atividade educativa) quanto aos registros de enfermagem da instituição. Os dados observados antes e após a intervenção foram comparados, permitindo, dessa forma, avaliar a eficácia da intervenção sobre a variável de interesse (registros de enfermagem).

Na primeira etapa do estudo, foi, inicialmente, realizada uma avaliação descritiva que apontou a situação dos registros de enfermagem da instituição ${ }^{(12)}$. Para a referida investigação, foi elaborado um roteiro com base na legislação sobre registros de enfermagem ${ }^{(7,13)}$ no qual foram avaliados os seguintes itens: uso de impresso próprio; identificação completa do paciente; data; hora; identificação do profissional responsável por meio de assinatura e carimbo ou inserção manual das informações de identificação profissional conforme legislação vigente; rasuras; apontamento de erros sem ocultar original; uso de abreviaturas padronizadas; letra legível e ortografia.

Cabe esclarecer que os erros ocorridos na elaboração dos registros de enfermagem devem ser apontados utilizando-se do termo "digo" seguido da informação corrigida, sendo proibido o emprego de meios que possam inviabilizar a leitura do erro ocorrido, como riscar o documento várias vezes, o uso de corretivo ou de borracha, entre outros. Em relação à padronização de abreviaturas, esta não havia sido implantada no período anterior à intervenção. Assim, para esse momento, foram consideradas somente as abreviaturas que constam no Sistema Internacional de Unidades ${ }^{(14)}$. Esse sistema foi adotado oficialmente no território brasileiro por meio da Resolução n. ${ }^{\circ} 12$ de 1988 do Conselho Nacional de Metrologia, Normalização e Qualidade Industrial, como forma de padronização(15). 
A partir da constatação da necessidade de melhoria nos registros, foi estabelecida a parceria escola-serviço, a partir de um projeto de iniciação científica sobre registros de enfermagem e de um projeto de treinamento profissional vinculado à disciplina Administração em Enfermagem da Faculdade de Enfermagem da Universidade Federal de Juiz de Fora.

Foi elaborada a instrução de trabalho "Registros de Enfermagem" junto ao núcleo de Gestão da Qualidade do hospital, contendo em anexo a lista de padronização de abreviaturas institucional. Foi também construído um folder explicativo apontando aspectos favoráveis à realização do registro de enfermagem conforme legislação específica(7,13).

Inicialmente, foi realizado um encontro no auditório do hospital com convite aos profissionais de enfermagem para a exposição dialogada e a discussão sobre registros de enfermagem. Entretanto, houve baixa adesão, e percebeu-se que essa abordagem não seria eficaz para obter mudanças positivas em relação aos registros de enfermagem. Dessa forma, após uma reunião com a Direção de Enfermagem, a equipe responsável pela Educação Permanente do hospital e a enfermeira do Núcleo de Gestão da Qualidade, decidiu-se por realizar encontros nos postos de enfermagem.

A intervenção constituiu-se, então, de encontros realizados com os profissionais de enfermagem nos postos de enfermagem da instituição, durante o horário de trabalho, em todos os turnos e setores. O cronograma de encontros foi construído a fim de englobar o maior quantitativo de profissionais considerando-se as atividades diárias, a rotina e o perfil de cada unidade, e o horário de descanso e/ ou refeições das equipes. Nos encontros, era apresentada e disponibilizada uma cópia da instrução de trabalho e distribuído um fôlder para cada profissional participante. Ressaltava-se a importância e a necessidade do cumprimento da legislação referente aos registros e a responsabilidade individual pela qualidade e pela disponibilidade das informações no prontuário do paciente.

A fim de avaliar os resultados da intervenção, foi replicado o estudo dos registros de enfermagem da instituição ${ }^{(12)}$ utilizando-se do mesmo instrumento de coleta de dados. Dessa forma, foram avaliados os registros de enfermagem de prontuários arquivados no Serviço de Arquivo Médico e Estatística.

Critérios de inclusão: registros de profissionais de enfermagem realizados a partir de $1^{\circ} \mathrm{de}$ fevereiro de 2013. Esse critério foi estabelecido considerando-se o período de implementação da instrução de trabalho "Registros de Enfermagem" contendo a lista de padronização de abreviaturas da instituição, bem como o período de realização da intervenção. Foram incluídos no estudo os seis primeiros prontuários (a partir de $1^{\circ}$ de fevereiro de 2013 ) das unidades: clínica feminina, clínica masculina, cirurgia feminina, cirurgia masculina, pediatria e unidade de terapia intensiva. Para a seleção dos prontuários, foi considerado o período de internação maior ou igual a 10 dias. Esse critério foi adotado a fim de permitir a avaliação dos registros dos diferentes turnos e jornadas de trabalho que coexistem na instituição (diaristas, plantonistas 12/36 horas diurno e noturno, plantonistas $12 / 60$ horas diurno e noturno).

Critérios de exclusão: registros de outros profissionais da equipe multidisciplinar e de acadêmicos, estagiários ou residentes.

Os dados foram coletados entre março e maio de 2013 e, após a coleta, foram transportados para uma base de dados eletrônica. Foram construídas tabelas de frequência das variáveis. Para verificar a diferença entre cada variável antes e após o treinamento por turno, categoria 
profissional, setor e número de infrações, foram construídas tabelas de contingência. O número de infrações por registro foi contabilizado considerando-se os itens de caracterização dos registros que constavam no instrumento de coleta de dados. Assim, se havia não conformidade em relação a determinado item, era computada uma infração, e assim sucessivamente.

A significância das associações foi verificada por meio da aplicação do teste qui-quadrado de Pearson. Foram considerados significantes valores de $p$ inferiores a 0,05 . Os dados foram digitados e analisados com o auxílio do programa Statistical Package for the Social Sciences (SPSS) versão 15.0 .

A presente investigação seguiu as recomendações éticas e legais vigentes, sendo apreciada e aprovada pelo Comitê de Ética em Pesquisa da instituição sob parecer número 133.384 antes do início da coleta dos dados.

\section{RESULTADOS}

Foram avaliados 826 registros de enfermagem, sendo 465 antes da intervenção; e 361, após a intervenção. $\mathrm{O}$ maior quantitativo de registros foi identificado na Unidade de Terapia Intensiva, entre os técnicos de enfermagem e no período noturno. O perfil dos registros de enfermagem antes e após a intervenção é apresentado na Tabela 1.

Tabela 1 - Perfil dos registros de enfermagem por setor, categoria profissional, turno e número de infrações antes e depois do treinamento. Juiz de Fora, 2013.

\begin{tabular}{lcccc}
\hline & \multicolumn{2}{c}{$\begin{array}{c}\text { Antes do Trei- } \\
\text { namento }\end{array}$} & \multicolumn{2}{c}{$\begin{array}{c}\text { Depois do } \\
\text { Treinamento }\end{array}$} \\
\cline { 2 - 5 } & $\mathbf{n}(\mathbf{4 6 5 )}$ & $\%$ & $\mathbf{n ~ ( 3 6 1 )}$ & $\%$ \\
\hline Setor (p=0,004) & & & & \\
\hline Terapia Intensiva & 190 & 40,8 & 147 & 40,7 \\
Clínica Masculina & 39 & 8,4 & 51 & 14,1
\end{tabular}

\begin{tabular}{|c|c|c|c|c|}
\hline Clínica Feminina & 38 & 8,2 & 29 & 8,0 \\
\hline Pediatria & 64 & 13,8 & 32 & 8,9 \\
\hline Cirurgia Masculina & 86 & 18,5 & 48 & 13,3 \\
\hline Cirurgia Feminina & 48 & 10,3 & 54 & 15,0 \\
\hline \multicolumn{5}{|c|}{ Categoria profissional $(p=0,008)$} \\
\hline Enfermeiro & 207 & 44,5 & 157 & 43,5 \\
\hline $\begin{array}{l}\text { Técnico de Enfer- } \\
\text { magem }\end{array}$ & 236 & 50,8 & 167 & 46,3 \\
\hline $\begin{array}{l}\text { Auxiliar de Enfer- } \\
\text { magem }\end{array}$ & 22 & 4,7 & 37 & 10,2 \\
\hline \multicolumn{5}{|l|}{ Turno $(p=0,034)$} \\
\hline Diurno & 225 & 48,4 & 148 & 41,0 \\
\hline Noturno & 240 & 51,6 & 213 & 59,0 \\
\hline \multicolumn{5}{|c|}{ Número de Infrações $(p<0,001)$} \\
\hline Nenhuma & 83 & 17,8 & 55 & 15,2 \\
\hline Uma ou duas & 243 & 52,3 & 266 & 73,7 \\
\hline Três ou mais & 139 & 29,9 & 40 & 11,1 \\
\hline
\end{tabular}

Legenda: Valores de $p$ obtidos por meio do teste qui-quadrado de Pearson. Fonte: autoria própria.

Em relação aos setores, foi identificado um aumento no percentual de registros na clínica masculina e na cirurgia feminina. Resultado oposto foi obtido nos setores pediatria e cirurgia masculina $(p=0,004)$. Na Unidade de Terapia Intensiva, o quantitativo de registros sofreu pouca variação.

A categoria profissional que mais foi influenciada pela intervenção foi a dos auxiliares de enfermagem, apresentando um aumento de mais de cinco pontos percentuais $(p=0,008)$. A diferença entre o quantitativo de registros entre os turnos diurno e noturno foi pequena antes da intervenção, porém essa diferença ficou mais distinta após o treinamento, com predomínio de registros realizados no turno noturno $(p=0,034)$. Outra mudança positiva obtida após a intervenção refere-se à diminuição do quantitativo de registros com três infrações ou mais $(p<0,001)$.

Ao considerar os itens que garantem a validade dos registros de enfermagem, foi possível observar melhorias significativas na presença de: data, hora, assinatura e carimbo. As características dos registros de enfermagem antes e após o treinamento são apresentadas no Gráfico 1. 
Gráfico 1 - Características dos registros de enfermagem antes e após o treinamento. Juiz de Fora, 2013.

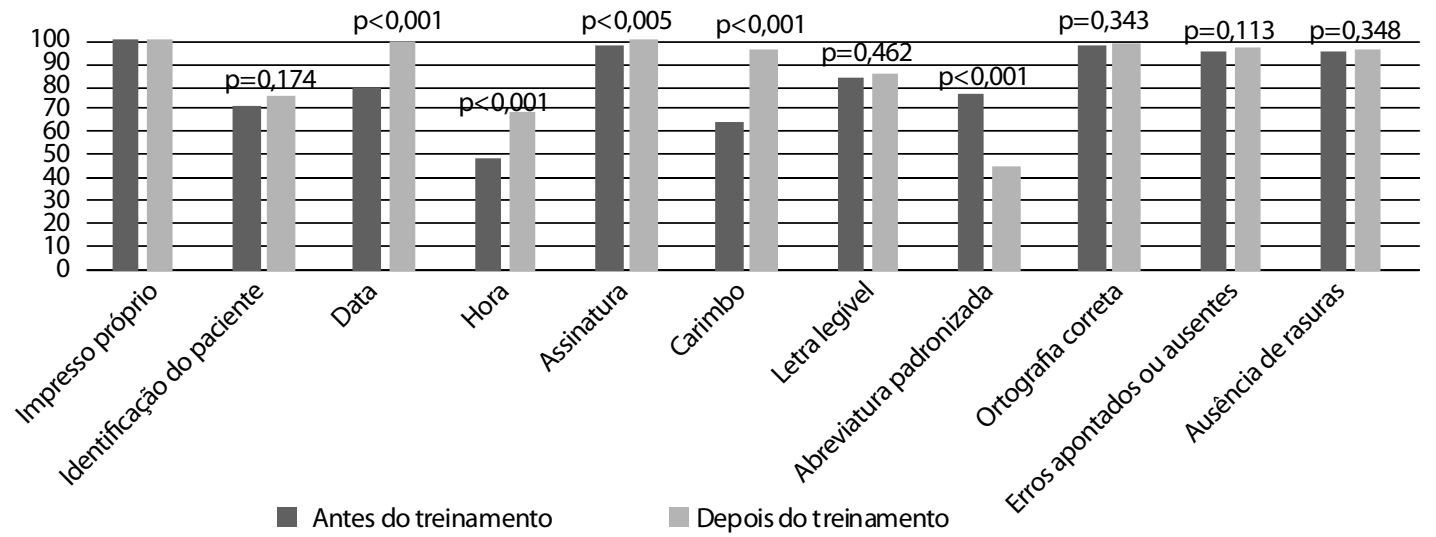

Legenda: Valores de p obtidos por meio do teste qui-quadrado de Pearson. Fonte: autoria própria.

Destaca-se que alguns itens se apresentavam em conformidade na quase totalidade dos registros observados nas avaliações antes e após a intervenção, como: ortografia correta, erros devidamente apontados ou ausentes e ausência de rasuras. Todos os registros de enfermagem foram realizados em impresso próprio antes e após o treinamento.

\section{DISCUSSÃO}

Os registros de enfermagem são um reflexo da práxis profissional e retratam a competência e o envolvimento dos profissionais no exercício de suas atividades. Assim, buscou-se nessa investigação avaliar os registros da equipe de enfermagem de um hospital de ensino em relação ao setor da instituição, ao turno de trabalho, à categoria profissional e ao número de infrações por registro antes e após intervenção educativa quanto ao cumprimento da legislação específica.

Durante o período de coleta de dados, a instituição enfrentava um período de transição de gestão, assim como outros hospitais de ensino. Mudanças nos regimes, contratação e déficit no quadro de pessoal eram então vivenciados. Além disso, a presença de profissionais com diferentes tipos de vínculos, alguns considerados precários ou desregulamentados ${ }^{(16)}$, impõe uma variação no ritmo de trabalho com reflexo nos registros realizados pelos profissionais de enfermagem. As diferenças de vínculo, jornada e remuneração que coexistem em um mesmo ambiente de trabalho podem produzir desmotivação e influenciar no rendimento dos profissionais $^{(17)}$. Dessa forma, podem ser justificadas as variações nos quantitativos de registros de enfermagem antes e após a intervenção entre os diferentes setores.

A maior concentração de registros na Unidade de Terapia Intensiva pode ser atribuída ao perfil dos pacientes que são admitidos nesse setor: pacientes com necessidades de cuidados intensivos, abrangendo quadros variados que podem incluir instabilidade hemodinâmica, dependência contínua de equipamentos de suporte para a vida, uso de drogas vasoativas, necessidade de monitorização contínua de sinais vitais, entre outras condições que exigem atenção contínua e especializada da equipe multiprofissional. Nesse ambiente, os registros de enfermagem podem ser mais frequentes devido às variações que podem sofrer as neces- 
sidades dos pacientes. Além disso, a Unidade de Terapia Intensiva é o único setor que possui a Sistematização da Assistência de Enfermagem implantada de forma completa desde o ano 2000 na instituição, o que implica novamente o maior empenho da equipe em registrar todos os passos da assistência prestada ao paciente ${ }^{(18)}$.

O maior quantitativo de registros foi realizado pelos técnicos de enfermagem, fato que pode ser explicado por serem a maior fração da equipe de enfermagem na instituição. Entretanto, os auxiliares de enfermagem foram os profissionais que mais aumentaram o quantitativo de registros após a intervenção. $\mathrm{O}$ grupo de auxiliares de enfermagem da instituição é composto exclusivamente por profissionais que fazem parte do quadro de profissionais efetivo, não sendo admitida contratação de novos profissionais com essa formação. Acredita-se que o vínculo efetivo pode ser uma influência positiva na adesão às recomendações, visto que a situação do profissional é "permanente" em relação ao local de trabalho e mudanças consideradas positivas nas atividades desenvolvidas cotidianamente podem refletir diretamente sobre seu bem-estar e sua satisfação no trabalho ${ }^{(17)}$.

O turno noturno foi responsável por um maior quantitativo de registros antes e após a intervenção. Esse fato pode ser explicado pela dinâmica de funcionamento do serviço hospitalar, que concentra no período diurno atividades diversas como exames, cirurgias, medicações, higiene do paciente, visitas de familiares, visitas médicas e de outros profissionais de saúde, entre outras. Além disso, por se tratar de uma instituição de ensino, há uma concentração das atividades de ensino também no período diurno, incluindo a realização de registros de enfermagem por acadêmicos, os quais não foram contemplados nessa investigação. Assim, pode-se justificar o fato de um volume maior de registros de enfermagem ter sido realizado pelos profissionais que desenvolvem seu trabalho no período noturno.

Identificou-se que os registros com três ou mais infrações apresentaram redução, demonstrando que a intervenção trouxe resultados positivos. Atividades educativas podem melhorar a qualidade dos registros de enfermagem ${ }^{(2)}$, permitindo que estes sejam válidos e confiáveis.

Ao se discutir os itens avaliados em cada registro, destacaram-se os resultados obtidos em relação à presença de data, hora, assinatura e carimbo. Durante a realização das atividades educativas de intervenção, esses itens foram muito discutidos, pois são itens que os profissionais sabem que devem ser inseridos, mas que por pressa ou descuido acabam sendo esquecidos $^{(10)}$. Após a intervenção, os profissionais ficaram mais atentos a esses itens.

A presença de data e hora permite a identificação do momento exato em que ocorreu o fato descrito com o paciente, favorecendo a continuidade da assistência fundamentada em dados precisos e organizados ${ }^{(2,10)}$.

A identificação do profissional responsável pelo registro por meio de assinatura e carimbo contendo dados profissionais é essencial, considerando-se que se trata de uma exigência legal ${ }^{(13)}$. Esse fato foi ressaltado durante os encontros, apontando resolução específica, o que conferiu maior credibilidade à informação apresentada e fundamentou as recomendações oferecidas. Além disso, a ausência de identificação do responsável pelo registro inviabiliza sua validade legal ${ }^{(19)}$, o que implica a ausência de respaldo em caso de questões judiciais. Ainda assim, alguns profissionais não realizam os registros de enfermagem com a qualidade que é esperada, apesar de considerarem o registro como parte essencial do seu trabalho ${ }^{(6,20)}$.

O único item que sofreu redução após a intervenção foi a presença de abreviaturas padronizadas. Tal fato pode ser explicado pela recente 
implementação da lista de padronização de abreviaturas institucional, a qual foi disponibilizada anexa à instrução de trabalho por ocasião da intervenção. O uso de abreviaturas padronizadas é essencial para a comunicação efetiva entre os profissionais de saúde, permitindo a adequada compreensão do que foi registrado. Em oposição, se são utilizadas abreviaturas não padronizadas, podem ocorrer ruídos comunicacionais com potencial prejuízo à segurança do paciente e à qualidade da assistência oferecida ${ }^{(8)}$.

A documentação de enfermagem completa, correta e fidedigna favorece a comunicação entre os membros da equipe de enfermagem e da equipe multiprofissional, promove o cuidado de enfermagem, facilita atingir objetivos profissionais, atende requisitos legais, auxilia a qualidade da assistência e demonstra credibilidade. De forma contrária, registros incompletos podem comprometer a segurança do paciente e a qualidade da assistência, com possíveis implicações profissionais e organizacionais(10).

Os bons resultados obtidos com esta investigação evidenciam quão importante é para o enfermeiro assumir seu papel de liderança junto à equipe de enfermagem e promover um dos pilares de seu processo de trabalho, que é o ensinar. Atividades educativas representam possibilidades de transformação, de visualização e de valorização dos conhecimentos dos sujeitos envolvidos, criando um movimento dinâmico do fazer e do refazer-se e tornando os trabalhadores mais comprometidos com o seu processo de trabalho(2).

Os profissionais de enfermagem reconhecem a importância do registro, entretanto, a documentação das ações desenvolvidas junto ao paciente é frequentemente deixada em segundo plano. Algumas razões são apresentadas para justificar esse fato, entre elas a falta de tempo decorrente da excessiva carga de trabalho e a dinâmica de funcionamento das unidades assistenciais nas quais atividades paralelas à assistência ao paciente são impostas aos profissionais, tomando-lhes o tempo que poderia ser dedicado ao registro do cuidado prestado ao paciente/família( ${ }^{(6)}$. Ressalta-se que é função do enfermeiro proporcionar à sua equipe o conhecimento necessário e buscar os meios para que os profissionais possam realizar o registro de enfermagem.

\section{CONCLUSÃO}

A avaliação dos registros de enfermagem antes e após uma intervenção educativa permitiu identificar que tal atividade promoveu mudanças no perfil por setor, turno de trabalho, categoria profissional e número de infrações por registro, bem como nas características dos registros de enfermagem, destacando-se os itens presença de data, hora, assinatura e carimbo.

Acredita-se que o uso de tecnologias simples e de baixo custo, como intervenções educativas, pode impactar no desempenho das equipes de enfermagem, promovendo transformações positivas na realidade de trabalho. $A$ associação ensino-serviço é uma parceria desejável, pois pode proporcionar crescimento bilateral, favorecendo o desenvolvimento da profissão e a qualidade dos serviços oferecidos à comunidade.

Para a promoção constante de melhorias nos registros de enfermagem, sugere-se a avaliação rotineira destes por meio de auditorias internas, as quais podem revelar itens que precisam ser melhor trabalhados com as equipes.

A informatização pode ser uma estratégia capaz de solucionar alguns dos problemas apontados nesta investigação, como a letra ilegível, a presença de data e hora, a identificação completa do paciente e a presença de rasuras. 
O uso de softwares específicos também pode ser eficiente na redução do uso de abreviaturas, quando ferramentas de autopreenchimento são adicionadas.

Uma das limitações da presente investigação é ter sido restrita a uma única instituição e terem sido avaliados somente itens de validade legal e estética dos registros de enfermagem. Investigações que considerem o conteúdo dos registros e possam avaliar os registros da equipe multiprofissional devem ser futuramente realizadas.

\section{REFERÊNCIAS}

1. 1. Kim H, Dykes PC, Thomas D, Winfield LA, Rocha RA. A closer look at nursing documentation on paper forms: Preparation for computerizing a nursing documentation system. Comput Biol Med. 2011 Apr [cited 2015 Nov 28];41(4):182-9. Available from: http://www. computersinbiologyandmedicine.com/article/ S0010-4825\%2810\%2900121-6/pdf.

2. 2. Wang N, Hailey D, Yu P. Quality of nursing documentation and approaches to its evaluation: a mixed-method systematic review. J Adv Nurs. 2011 Sep [cited 2015 Aug 19];67(9):1858-75. Available from: https://www.ksa.ch/intranetEvents/ Attachments/1626/Wang_2011_quality_tools_documentation.pdf.

3. 3. Barreto PA, Braga ALS, Andrade M. Evaluation of completeness of dengue records: exploratory study of compulsory notices. Online braz j nurs [internet]. 2012 Dec [cited 2016 Jan 29]; 11(3):829-47. Available from: http://www.objnursing.uff.br/index.php/nursing/article/view/3920.

4. 4. Galvão MCB; Ricarte ILM. Prontuário do Paciente. Rio de Janeiro: Guanabara Koogan, 2012.

5. 5. Conselho Federal de Enfermagem. Resolução COFEN 311, de 08 de fevereiro de 2007. Aprova a reformulação do código de ética dos profissionais de enfermagem. [internet]. Brasília: COFEN; 2007. [cited 2015 Sep 10]. Available from: http://www. cofen.gov.br/resoluo-cofen-3112007_4345.html.
6. 6. Blake-Mowatt C, Lindo JLM, Bennett J. Evaluation of registered nurses' knowledge and practice of documentation at a Jamaican hospital. Int Nurs Rev. [internet] 2013 Sep [cited 2015 Dec 12];60(3):328-334. Available from: http://onlinelibrary.wiley.com/doi/10.1111/inr.12040/epdf.

7. 7. Conselho Regional de Enfermagem de Minas Gerais. Deliberação COREN-MG 135/2000 de 10 de outubro de 2000. Normatiza no Estado de Minas Gerais os princípios gerais que constituem a documentação de Enfermagem. [internet]. Belo Horizonte: COREN-MG; 2000. [cited 2015 Sep 10]. Available from: http://www.corenmg.gov.br/ anexos/legislacao_normas_pb.pdf.

8. 8. Jefferies D. Communicate with clarity: Correct use of abbreviations and phrases in nursing records is vital for care efficiency and safety. Nurs Stand. [internet] 2012 Jun [cited 2015 Oct 17]; 26(40):62-3. Available from: http://go.galegroup. com/ps/i.do?id=GALE|A295170938\&sid=google Scholar\&v=2.1\&it=r\&linkaccess $=$ fulltext $\& i s s n=0$ $0296570 \& p=A O N E \& s w=w \& a u t h C o u n t=1 \& u=c a$ pes\&selfRedirect=true.

9. 9. Santos JLG, Pestana AL, Guerrero P, Meirelles BSH, Erdmann AL. Práticas de enfermeiros na gerência do cuidado em enfermagem e saúde: revisão integrativa. Rev. bras. enferm. [Internet]. 2013 Apr [cited 2016 Feb 23];66(2):257-263. Available from: http://www.scielo.br/pdf/reben/ v66n2/16.pdf.

10. 10. Blair W, Smith B. Nursing documentation: Frameworks and barriers. Contemp Nurse. [internet] 2012 Jun [cited 2015 Aug 28]; 41 (2):160-8. Available from: http://go.galegroup.com/ps/i.do ?id=GALE $\mid$ A298057135\&sid=googleScholar $\& v=$ $2.1 \& i t=r \&$ linkaccess $=$ fulltext\&issn $=10376178 \&$ $\mathrm{p}=\mathrm{AONE} \& \mathrm{sw}=\mathrm{w} \&$ authCount $=1 \& \mathrm{u}=$ capes $\&$ selfR edirect=true.

11. 11. Agência Nacional de Vigilância Sanitária. Assistência segura: uma reflexão teórica aplicada à prática. [internet]. Brasília: ANVISA; 2013. [cited 2015 Dec 28]. Available from: http://www20. anvisa.gov.br/segurancadopaciente/images/documentos/livros/Livro1-Assistencia_Segura.pdf.

12. 12. Barboza RCP. Avaliação da qualidade das anotações de enfermagem em um Hospital Universitário. Trabalho de Conclusão de Curso. Universidade Federal de Juiz de Fora, 2011. 
13. 13. Conselho Federal de Enfermagem. Resolução COFEN 191 de 31 de maio de 1996. Dispõe sobre a forma de anotação e o uso do número de inscrição ou da autorização, pelo pessoal de Enfermagem. [internet]. Brasília: COFEN; 1996. [cited 2015 Sept 10] Available from: http://www. cofen.gov.br/resoluo-cofen-1911996-revogou-resoluo-cofen-1751994_4250.html.

14. 14. Instituto Nacional de Metrologia, Qualidade e Tecnologia (INMETRO). Sistema Internacional de Unidades: SI. [internet]. Duque de Caxias, RJ: INMETRO/CICMA/SEPIN; 2012. [cited 2015 Aug 05]. Available from: http://www.inmetro.gov. br/noticias/conteudo/sistema-internacional-unidades.pdf.

15. 15. Conselho Nacional de Metrologia, Normalização e Qualidade Industrial - CONMETRO. Resolução no 11, de 12 de outubro de 1988. [internet]. Brasília: CONMETRO; 1988. [cited 2015 Aug 31]. Available from: http://www.inmetro.gov.br/resc/ pdf/RESC000114.pdf.

16. 16. Sodré F, Littike D, Drago LMB, Perim MCM. Empresa Brasileira de Serviços Hospitalares: um novo modelo de gestão? Serv. Soc. Soc. [internet] 2013 Apr-Jun; 114:365-80. Available from: http:// www.scielo.br/pdf/sssoc/n114/n114a09.pdf.

17. 17. Ribeiro AC, Souza JF, Silva JL. Lack of employment security in the Sus in the perspective of Hospital nursing. Cogitare Enferm. [internet] 2014 Jul-Sep; [cited 2015 Nov 08];19(3):569-7. Available from: http://ojs.c3sl.ufpr.br/ojs2/index. php/cogitare/article/view/33034/23248.

18. 18. Conselho Federal de Enfermagem. Resolução COFEN-358/2009. Dispõe sobre a Sistematização da Assistência de Enfermagem e a implementação do Processo de Enfermagem em ambientes, públicos ou privados, em que ocorre o cuidado profissional de Enfermagem, e dá outras providências. [internet]. Brasília: COFEN; 2009. [cited 2015 Sept 10]. Available from: http://www.cofen. gov.br/resoluo-cofen-3582009_4384.html.
19. 19. Brasil. Código Processo Civil. Lei n 5.869, de 11 de janeiro de 1973. [Internet]. Brasília: Presidência da República; 1973. [cited 20 Jan 2016]. Available from: http://presrepublica.jusbrasil. com.br/legislacao/91735/codigo-processo-civil-lei-5869-73.

20. 20. Azevêdo LMN, Oliveira AG, Malveira FAS, Valença CN, Costa EO, Germano RM. A visão da equipe de enfermagem sobre seus registros. Rev Rene. [internet] 2012 [cited 2015 Oct 30]; 13(1):64-73. Available from: http://www.revistarene.ufc.br/revista/index.php/revista/article/ view/18/14.

Todos os autores participaram das fases dessa publicação em uma ou mais das etapas a seguir, de acordo com as recomendações do International Committe of Medical Journal Editors (ICMJE, 2013): (a) participação substancial na concepção ou na confecção do manuscrito ou da coleta, da análise ou da interpretação dos dados; (b) elaboração do trabalho ou realização de revisão crítica do conteúdo intelectual; (c) aprovação da versão submetida. Todos os autores declaram para os devidos fins que são de suas responsabilidades o conteúdo relacionado a todos os aspectos do manuscrito submetido ao OBJN. Garantem que as questões relacionadas à exatidão ou à integridade de qualquer parte do artigo foram devidamente investigadas e resolvidas. Eximindo, portanto o OBJN de qualquer participação solidária em eventuais imbróglios sobre a matéria em apreço. Todos os autores declaram que não possuem conflito de interesses, seja de ordem financeira, seja de relacionamento, que influencie a redação e/ou a interpretação dos achados. Essa declaração foi assinada digitalmente por todos os autores conforme recomendação do ICMJE, cujo modelo está disponível em <http://www. objnursing.uff.br/normas/DUDE_final_13-06-2013.pdf>.

Recebido: 30/01/2016

Revisado: 29/06/2016

Aprovado: 03/07/2016 